DESY 06-158

\title{
Dark Matter from Gaugino Mediation
}

\author{
Wilfried Buchmüller ${ }^{\mathrm{a}}$, Laura Covi ${ }^{\mathrm{b}}$, Jörn Kersten ${ }^{\mathrm{c}}$ \\ and Kai Schmidt-Hoberg ${ }^{\mathrm{d}}$ \\ Deutsches Elektronen-Synchrotron DESY, 22603 Hamburg, Germany
}

\begin{abstract}
We study dark matter (DM) for gaugino-mediated supersymmetry breaking and compact dimensions of order the grand unification scale. Higgs fields are bulk fields, and in general their masses differ from those of squarks and sleptons at the unification scale. As a consequence, at different points in parameter space, the gravitino, a neutralino or a scalar lepton can be the lightest (LSP) or nextto-lightest (NLSP) superparticle. We investigate the constraints from primordial nucleosynthesis on the different scenarios. While neutralino DM and gravitino DM with a $\widetilde{\nu}$ NLSP are consistent for a wide range of parameters, gravitino DM with a $\widetilde{\tau}$ NLSP is strongly constrained. Gravitino DM with a $\chi^{0}$ NLSP is excluded.
\end{abstract}

\footnotetext{
${ }^{a}$ Email: wilfried.buchmueller@desy.de

${ }^{b}$ Email: laura.covi@desy.de

${ }^{c}$ Email: joern.kersten@desy.de

${ }^{\mathrm{d}}$ Email: kai.schmidt.hoberg@desy.de
} 


\section{Introduction}

Gaugino mediation [1,2] is an attractive way to introduce supersymmetry (SUSY) breaking in higher-dimensional theories with four-dimensional branes. For squarks and sleptons which are confined to these branes this yields no-scale boundary conditions [3], whereas gauginos and Higgs fields acquire soft SUSY breaking masses at tree-level, since they are bulk fields.

Varying the boundary conditions for the Higgs fields at the GUT scale, we found in [4] that, apart from the gravitino, a neutralino or a scalar lepton, $\widetilde{\tau}$ or $\widetilde{\nu}$, can be the lightest superparticle (LSP). Since a scalar lepton is excluded as LSP [5, 6], it can only be the next-to-lightest superparticle (NLSP) with the gravitino as LSP, which is consistent with the lower bound on the gravitino mass in gaugino mediation [7]. One then obtains the $\widetilde{G}-\widetilde{\tau}$ and the $\widetilde{G}-\widetilde{\nu}$ scenarios with $\widetilde{\tau}$ and $\widetilde{\nu}$ as NLSP, respectively.

The $\widetilde{G}-\widetilde{\tau}$ scenario is particularly interesting, since it may allow to determine the gravitino mass and spin at colliders [8]. It is well known, however, to be strongly constrained by primordial nucleosynthesis (BBN) $[9,10,11]$. In the following we therefore study the impact of such constraints on the $\widetilde{G}-\widetilde{\tau}$ scenario in gaugino mediation where the gravitino mass is bounded from below, leading to very long-lived $\widetilde{\tau}$ leptons, and compare it with the $\widetilde{G}-\widetilde{\nu}$ scenario.

The decays of a neutralino NLSP into gravitino and photon or $Z$ boson, decaying further into hadrons, make this scenario incompatible with BBN. On the other hand, we find that a neutralino LSP as dominant component of dark matter is a viable possibility in gaugino mediation.

The paper is organised as follows. In Section 2 and 3 we briefly recall the boundary conditions for gaugino mediation and the BBN constraints on NLSP abundances, respectively. Section 4 deals with neutralino dark matter, and in Section 5 we discuss gravitino dark matter with slepton NLSPs. Our results are summarised in Section 6.

\section{Superparticle Masses from Gaugino Mediation}

Consider a theory with $D$ space-time dimensions and four-dimensional branes located at different positions in the $D-4$ compact spatial dimensions. In models with gaugino mediation $[1,2]$, the gauge superfields live in the bulk, while the chiral superfield $S$ responsible for SUSY breaking is localised on one of the four-dimensional branes. The Higgs fields can live in the bulk as well.

A vacuum expectation value $F_{S}$ for the $F$-term of $S$ breaks SUSY and leads to a nonvanishing gaugino mass $m_{1 / 2}$ as well as a gravitino mass $m_{3 / 2}$ at the compactification scale $M_{C}$, which we assume to be of order the unification scale $M_{\mathrm{GUT}}$. Like the gaugino masses, also the soft Higgs masses $m_{\tilde{h}_{i}}^{2}$ and the parameters $\mu$ and $B \mu$ are generated from 
non-renormalisable couplings with the brane field $S$. Here $\tilde{h}_{1}$ is the Higgs which couples to the down-type quarks, whereas $\tilde{h}_{2}$ is the up-type Higgs. Neglecting small corrections to the scalar masses from gaugino loops as well as corrections to the gauge couplings from brane-localised terms breaking the unified gauge symmetry, one obtains the boundary conditions of gaugino mediation with bulk Higgs fields at the compactification scale [2]:

$$
\begin{aligned}
& g_{1}=g_{2}=g_{3}=g \simeq 1 / \sqrt{2} \\
& M_{1}=M_{2}=M_{3}=m_{1 / 2}, \\
& m_{\tilde{\phi}_{\mathrm{L}}}^{2}=m_{\tilde{\phi}_{\mathrm{R}}}^{2}=0 \quad \text { for all squarks and sleptons } \tilde{\phi} \\
& A_{\tilde{\phi}}=0 \quad \text { for all squarks and sleptons } \tilde{\phi} \\
& \mu, B \mu, m_{\tilde{h}_{i}}^{2} \neq 0 \quad(i=1,2)
\end{aligned}
$$

where the GUT charge normalisation is used for $g_{1}$. If the Higgs fields are localised on a brane, one has $m_{\tilde{h}_{i}}^{2}=B \mu=0$, which is a special case of minimal supergravity.

The ranges of SUSY breaking parameters leading to a viable low-energy spectrum have been discussed in [4]. The spectrum is determined by the boundary conditions (1) and the renormalisation group equations. The model favours moderate values of $\tan \beta$ between about 10 and 25. Much smaller and larger values are in conflict with the LEP lower bounds on the Higgs mass and the $\widetilde{\tau}$ mass, respectively. The gaugino mass at the GUT scale cannot be far below $500 \mathrm{GeV}$ in order to satisfy the LEP bound on the Higgs mass. Typically, the lightest neutralino is bino-like with a mass of $200 \mathrm{GeV}$, and the gluino mass is about $1.2 \mathrm{TeV}$. Depending on $m_{\tilde{h}_{1}}^{2}$, either the right-handed or the left-handed sleptons can be lighter than the neutralinos. The corresponding region in parameter space grows with $\tan \beta$.

Gaugino mediation gives a lower bound on the gravitino mass. This bound depends on $m_{1 / 2}$, the number of space-time dimensions and the compactification scale [7]. Motivated by a six-dimensional orbifold GUT [12], we choose $D=6$ and $M_{C}=M_{\text {GUT }}$ leading to $m_{3 / 2} \gtrsim 0.1 \cdot m_{1 / 2} \gtrsim 50 \mathrm{GeV}$. As the lower limit on $m_{3 / 2}$ was derived using naïve dimensional analysis [13], it can well be relaxed by a factor of order one. We therefore also consider $m_{3 / 2}=10 \mathrm{GeV}$ as a conservative lower bound. Note that varying $D$ between 5 and 10, the lower bound ranges between $20 \mathrm{GeV}$ and $0.1 \mathrm{GeV}$.

\section{BBN Constraints on the Abundance of NLSPs}

Big Bang Nucleosynthesis starts about $100 \mathrm{~s}$ after the big bang at a temperature of about $0.1 \mathrm{MeV}$. In the scenario where the gravitino is the LSP, the NLSP decays considerably later. The decay products of such long-lived particles can alter the primordial light element abundances $[14,15,16]$. This leads to constraints on the released electromagnetic and hadronic energy. To a good approximation these constraints can be quantified by 
upper bounds on the product $\epsilon_{\mathrm{em}, \mathrm{had}} Y_{\mathrm{NLSP}}$. Here $\epsilon_{\mathrm{em} \text {,had }}$ is the average electromagnetic or hadronic energy emitted in a single NLSP decay and the abundance $Y_{\text {NLSP }}$ is given by the NLSP number density prior to decay divided by the total entropy density,

$$
Y_{\mathrm{NLSP}} \equiv \frac{n_{\mathrm{NLSP}}}{s}
$$

We determine this normalised NLSP number density numerically, assuming that the NLSP freezes out with its thermal relic density.

For our analysis we use the bounds compiled in Fig. 9 of [17] (see also Fig. 3 below), which were computed in the earlier studies $[14,15]$. These bounds assume an NLSP mass of $1 \mathrm{TeV}$, but since they are quite insensitive to this mass, we will use them here, too. Furthermore, they assume that there is no entropy production between the decoupling of the NLSP and the start of BBN. As there is still considerable uncertainty in the measurements of the primordial element abundances, [17] used two different data sets, giving "severe" and "conservative" limits. The severe limits are derived from

$$
\begin{aligned}
2.02 \cdot 10^{-5} & <\frac{n_{\mathrm{D}}}{n_{\mathrm{H}}}<3.66 \cdot 10^{-5}, \\
0.227 & <Y_{p}<0.249,
\end{aligned}
$$

where $n_{\mathrm{X}}$ is a primordial number density and $Y_{p}$ the primordial mass fraction of ${ }^{4} \mathrm{He}$. The relevant conservative limits are derived from

$$
\begin{aligned}
1.3 \cdot 10^{-5} & <\frac{n_{\mathrm{D}}}{n_{\mathrm{H}}}<5.3 \cdot 10^{-5}, \\
0.231 & <Y_{p}<0.253 .
\end{aligned}
$$

Note that although the upper bounds on the Deuterium abundance leading to the conservative and severe constraints differ by less than a factor of two, the resulting bounds on $\epsilon_{\mathrm{em}} Y_{\mathrm{NLSP}}$ differ by an order of magnitude.

The constraints on hadronic and electromagnetic energy release are assumed to be independent, although there can be cancellations between them in special cases. We consider points in parameter space violating the conservative limits to be "excluded", but points violating only the severe limits to be "disfavoured". The observed abundances of ${ }^{3} \mathrm{He},{ }^{6} \mathrm{Li}$ and ${ }^{7} \mathrm{Li}$ are not used, since they still suffer from large systematic uncertainties.

\section{Neutralino Dark Matter}

We are now in a position to determine the cosmologically allowed, disfavoured and excluded regions of the parameter space of models with gaugino-mediated supersymmetry breaking. Since moderate values of $\tan \beta$ are favoured, we consider the cases $\tan \beta=10$ and $\tan \beta=20$ in the following. As a benchmark point for our discussion we take 
the unified gaugino mass to be $m_{1 / 2}=500 \mathrm{GeV}$ and the supersymmetric Higgs mass parameter to be positive, sign $\mu>0$.

Both for the constraints from BBN and for those from the observed cold dark matter density, the abundance $Y_{(\mathrm{N}) \mathrm{LSP}}$ of the (N)LSP is essential. In this section we will discuss neutralino dark matter and then turn to gravitino dark matter with slepton NLSPs in the next section.

\subsection{Calculation of the Abundance}

We use micrOMEGAs 1.3.6 [18] to calculate the abundance and the energy density of the (N)LSP numerically. The superpartner spectrum is determined by SOFTSUSY 2.0.6 [19]. For the top quark pole mass, we use the latest best-fit value of $172.5 \mathrm{GeV}$ [20]. ${ }^{1}$

We first consider the case where a neutralino is lighter than all sleptons and squarks, so that it is an LSP or NLSP candidate. In the corresponding parameter space region for $\tan \beta=20$, we find numerically

$$
\begin{aligned}
2.6 \cdot 10^{-13} & \leq Y_{\chi} \leq 5.0 \cdot 10^{-12}, \\
83.0 \mathrm{GeV} & \leq m_{\chi} \leq 204 \mathrm{GeV}, \\
8.15 \cdot 10^{-3} & \leq \Omega_{\chi} h^{2} \leq 0.273 .
\end{aligned}
$$

For $\tan \beta=10$, the results are very similar, with a slightly larger maximal abundance of $8.7 \cdot 10^{-12}$. Here the relation between neutralino relic abundance $Y_{\chi}$ and energy density $\Omega_{\chi} h^{2}$ is given by

$$
Y_{\chi}=\frac{\Omega_{\chi} \rho_{c}}{s m_{\chi}} \simeq 3.64 \cdot 10^{-11}\left(\frac{100 \mathrm{GeV}}{m_{\chi}}\right) \Omega_{\chi} h^{2},
$$

with $\rho_{c}$ the critical density and $s$ the entropy density of the universe.

\subsection{Neutralino LSP}

Gaugino mediation provides only a lower bound on the mass of the gravitino. Therefore, it may well be quite heavy, and the lightest neutralino may be the LSP. In this case, decays of the long-lived gravitino threaten the success of BBN, which leads to an upper bound on the gravitino density and thus on the reheating temperature [14, 15]. The other superparticles decay into the LSP before the start of BBN and do not cause problems, unless LSP and NLSP are nearly degenerate. For example, if the NLSP is a stau, BBN constraints become potentially important for $m_{\widetilde{\tau}}-m_{\chi} \lesssim 100 \mathrm{MeV}$ [21]. We neglect this possibility, since the corresponding region in the parameter space is tiny.

\footnotetext{
${ }^{1}$ In addition, we use $m_{b}\left(m_{b}\right)=4.25 \mathrm{GeV}$ and $\alpha_{s}^{\mathrm{SM}} \overline{\mathrm{MS}}\left(M_{Z}\right)=0.1187$, the default values of SOFTSUSY. Some other SM parameters are hard-coded in micrOMEGAs, $\alpha_{\mathrm{em}}^{-1 \mathrm{SM}} \overline{\mathrm{MS}}\left(M_{Z}\right)=127.90896$, $G_{F}=1.16637 \cdot 10^{-5} \mathrm{GeV}^{-2}$, and $m_{\tau}=1.777 \mathrm{GeV}$.
} 
This leaves the observed dark matter density as the only constraint on the neutralino LSP scenario we have to consider. We use the $3 \sigma$ range given in $[22]^{2}$,

$$
0.106<\Omega_{\mathrm{DM}} h^{2}<0.123 .
$$

The upper limit excludes the white regions in Fig. 1. Since the dark matter could be made up of several components and since non-thermal production could be significant, we have two viable regions in parameter space. In the first one, the thermal neutralino relic density falls into the range (7) and hence this particle makes up all the dark matter. This region is shown in black in Fig. 1. There the bino contributes at least $75 \%(80 \%)$ to the lightest neutralino for $\tan \beta=10(\tan \beta=20)$. The missing $25 \%(20 \%)$ come from the two Higgsinos, while the wino component of $\sim 1 \%$ is negligible. On the left edge the lightest neutralino is a pure bino. The second viable region is shown in magenta (dark-gray) in the figure. Here the thermal neutralino density is smaller than the lower bound in Eq. (7) and hence only constitutes a part of the dark matter density. The lightest neutralino is almost a pure Higgsino at the right edge of the parameter space.

In most parts of the parameter space, some tuning is necessary if neutralinos are to make up all the dark matter. This is very similar to what has been found in other scenarios for SUSY breaking, for instance in mSUGRA (see for example [23]). In part, the reason is simply that the dark matter density has been measured rather accurately. For $\tan \beta=20$ and small $m_{\tilde{h}_{2}}^{2}$, the situation looks somewhat better. We are not aware of a simple physical explanation for this. Apparently, for $m_{\tilde{h}_{2}}^{2}<0.1 \mathrm{TeV}^{2}$ the maximum of $\Omega_{\chi}$ as a function of $m_{\tilde{h}_{1}}^{2}$ lies in the experimentally allowed region. Around the maximum, a change in $m_{\tilde{h}_{1}}^{2}$ leads only to a relatively small change in $\Omega_{\chi}$, so that the energy density remains in the favoured range in a rather broad strip of parameter space. For larger $m_{\tilde{h}_{2}}^{2}$, the maximum value of $\Omega_{\chi}$ is too large. Consequently, it depends rather sensitively on $m_{\tilde{h}_{1}}^{2}$ in the allowed region, and thus this region is narrow.

Let us finally comment on the direct detection of neutralino dark matter in our scenario. As in the general MSSM case, the detection cross-section is suppressed for a pure bino, since the Higgs and $Z$ exchange require a Higgsino component. In fact, for $\tan \beta=10, m_{\tilde{h}_{1}}^{2}=2.21 \mathrm{TeV}^{2}$ and $m_{\tilde{h}_{2}}^{2}=0$, one obtains $\sigma_{\chi p, n}=9 \cdot 10^{-13} \mathrm{nb}$ for the spin-independent cross-section per nucleon [24], whereas the present bound on this cross-section is of the order of $10^{-9} \mathrm{nb}$ [25]. The cross-section is larger in the region with a larger Higgsino component, where for $\tan \beta=10, m_{\tilde{h}_{1}}^{2}=2.76 \mathrm{TeV}^{2}, m_{\tilde{h}_{2}}^{2}=0.44 \mathrm{TeV}^{2}$, one obtains $\sigma_{\chi p, n}=4 \cdot 10^{-11} \mathrm{nb}[24]$. Although the cross-section is at least one order of magnitude below the present bounds, it could be reached by the next generation of dark matter experiments [26].

\footnotetext{
${ }^{2}$ The analysis (labelled "All Data - LYA") used the measurements of the CMB power spectrum (temperature and polarisation) by WMAP (3-year data) and other experiments, the SDSS and 2dF galaxy clustering analyses, the SDSS luminous red galaxy constraints on the acoustic peak, as well as the Gold and the SNLS supernovae samples.
} 

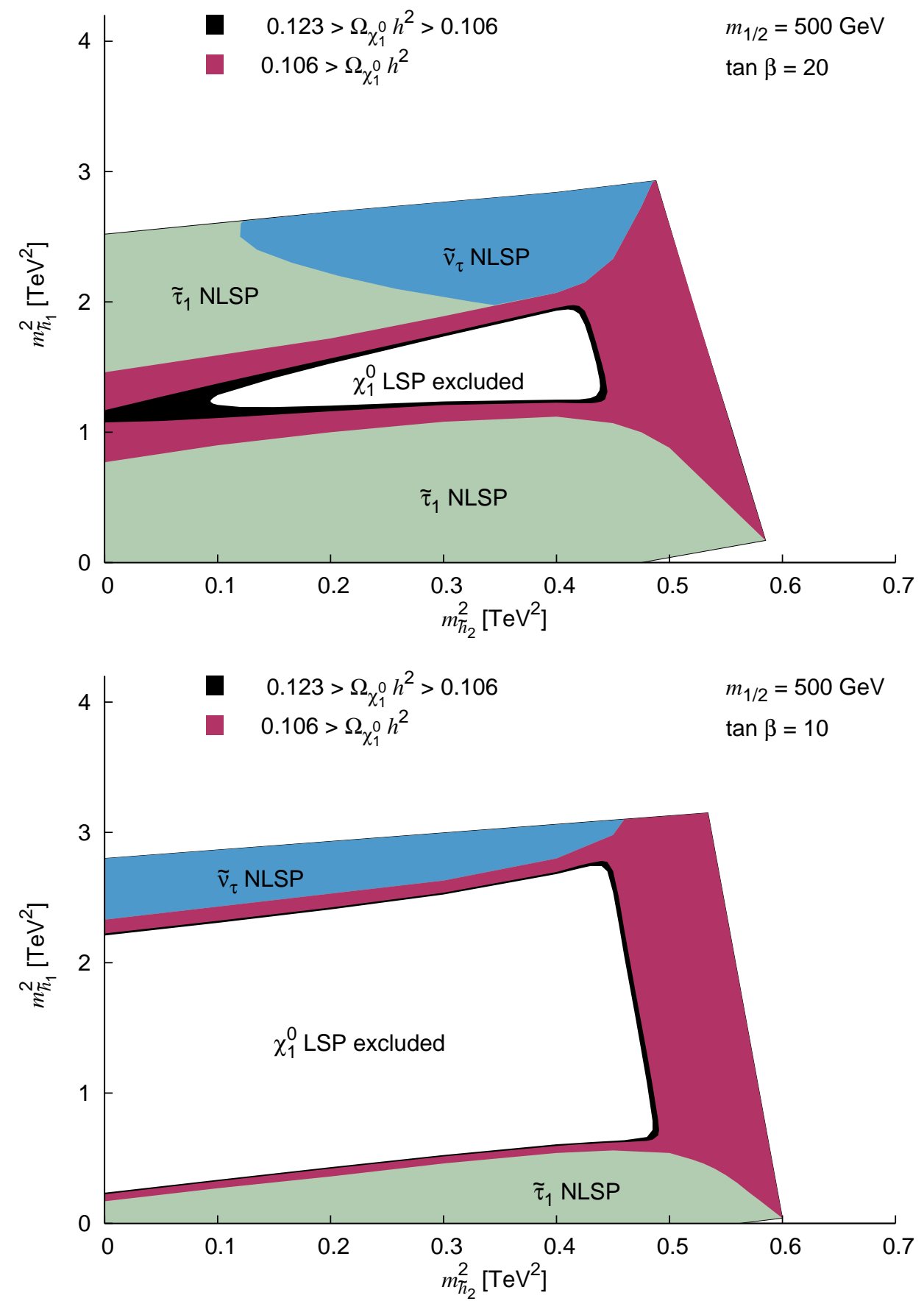

Figure 1: Allowed region for the soft Higgs masses for $m_{1 / 2}=500 \mathrm{GeV}$ and $\tan \beta=20(\tan \beta=10)$. A neutralino is lighter than all sleptons in the white, black and magenta (dark-gray) area. The upper limit on $\Omega_{\chi} h^{2}$ excludes the white region, whereas in the magenta (dark-gray) area $\Omega_{\chi} h^{2}$ is smaller than the observed cold dark matter density. The correct dark matter density is obtained in the black region. In the green (light-gray) and blue (medium-gray) areas a slepton is the NLSP. 


\subsection{Neutralino NLSP}

With a light gravitino, a scenario with a gravitino LSP and a neutralino NLSP is possible, too. However, it turns out that this is ruled out by the BBN constraints in gaugino mediation.

The region where $m_{\chi}>m_{3 / 2}+m_{Z}$ is certainly excluded by the hadronic BBN constraints for all gravitino masses we consider, since the two-body decay $\chi_{1}^{0} \rightarrow Z \widetilde{G}$ is possible and since the hadronic branching ratio of the $Z$ is large [10, 27]. However, the situation is less clear for lighter neutralinos when the two-body decay into real $Z$ bosons is not possible. For $m_{3 / 2}=50 \mathrm{GeV}$, this is the case for $m_{\chi}<141 \mathrm{GeV}$. The corresponding parameter space region lies at the right end of the allowed region, where $m_{\tilde{h}_{2}}^{2} \gtrsim 0.5 \mathrm{TeV}^{2}$. In this region, the $\mu$ parameter is rather small [4], so that there is significant mixing between the neutralinos. The Higgsino components of the lightest neutralino lead to a relatively large annihilation cross section and thus to a relatively small abundance. From Fig. 9 of [17] we can read off that the severe hadronic bound is never stronger than

$$
\epsilon_{\text {had }} Y_{\mathrm{NLSP}} \lesssim 5 \cdot 10^{-14} \mathrm{GeV}
$$

for any NLSP lifetime. With the estimate $\epsilon_{\text {had }}^{\chi} \simeq \frac{2}{3}\left(m_{\chi}-m_{3 / 2}\right) \cdot 10^{-3}$ from [10], we find

$$
1.0 \cdot 10^{-14} \mathrm{GeV} \leq \epsilon_{\text {had }}^{\chi} Y_{\chi} \leq 2.1 \cdot 10^{-14} \mathrm{GeV},
$$

which is well below the stringent hadronic bound (8). Considering electromagnetic energy release instead, we have

$$
\epsilon_{\mathrm{em}}^{\chi} \simeq \frac{m_{\chi}^{2}-m_{3 / 2}^{2}}{2 m_{\chi}}
$$

and

$$
\Gamma_{\chi}=\frac{\left|N_{11} \cos \theta_{W}+N_{12} \sin \theta_{W}\right|^{2} m_{\chi}^{5}}{48 \pi m_{3 / 2}^{2} M_{\mathrm{P}}^{2}}\left(1-\frac{m_{3 / 2}^{2}}{m_{\chi}^{2}}\right)^{3}\left(1+3 \frac{m_{3 / 2}^{2}}{m_{\chi}^{2}}\right)
$$

for the width of the dominant neutralino decay mode $\chi_{1}^{0} \rightarrow \gamma \widetilde{G}$, where $N_{1 i}$ are elements of the neutralino mixing matrix, so that e.g. $\left|N_{11}\right|^{2}$ is the bino fraction [10]. This leads to

$$
\begin{aligned}
1.1 \cdot 10^{-11} \mathrm{GeV} & \leq \epsilon_{\mathrm{em}}^{\chi} Y_{\chi} \leq 2.2 \cdot 10^{-11} \mathrm{GeV} \\
3.3 \cdot 10^{8} \mathrm{~s} & \leq \tau_{\chi} \leq 4.1 \cdot 10^{10} \mathrm{~s}
\end{aligned}
$$

for both $\tan \beta=10$ and $\tan \beta=20$. Comparing with the electromagnetic limits in Fig. 3, we see that even the conservative $\mathrm{BBN}$ bound is violated. This result remains true for $m_{3 / 2}=10 \mathrm{GeV}$. Thus, we conclude that a neutralino NLSP with a mass below $m_{Z}+m_{3 / 2}$ is excluded by the BBN constraints on electromagnetic energy release. Consequently, the lightest neutralino is not a viable NLSP candidate in gaugino mediation. 


\section{Gravitino Dark Matter with Slepton NLSPs}

\subsection{Lifetime of Slepton NLSPs}

The slepton decay rate is dominated by the two-body decay into lepton and gravitino,

$$
\Gamma_{\tilde{l}}^{2 \text {-body }}=\frac{m_{\tilde{l}}^{5}}{48 \pi m_{3 / 2}^{2} M_{\mathrm{P}}^{2}}\left(1-\frac{m_{3 / 2}^{2}}{m_{\tilde{l}}^{2}}\right)^{4},
$$

where $m_{\tilde{l}}$ is the slepton mass, $M_{\mathrm{P}}=2.4 \cdot 10^{18} \mathrm{GeV}$ is the reduced Planck mass, and where the lepton mass has been neglected. With a typical largest slepton mass of around $200 \mathrm{GeV}$ in the $\tilde{l}$ NLSP region and the smallest gravitino mass of $10 \mathrm{GeV}$ this leads to a lower bound on the slepton lifetime of

$$
\tau_{\tilde{l}} \gtrsim 1.8 \cdot 10^{5} \mathrm{~s}
$$

which is a time where the BBN constraints become stringent.

\subsection{Stau NLSP}

For both values of $\tan \beta$, imposing the lower bound from collider searches [6], we find

$$
86 \mathrm{GeV}<m_{\widetilde{\tau}} \leq 203 \mathrm{GeV}
$$

in the $\widetilde{\tau}$ NLSP region. The upper limit on the stau mass within this region only depends on the mass of the lightest neutralino and is therefore almost independent of $\tan \beta$. With $m_{3 / 2}=50 \mathrm{GeV}$, this mass range corresponds to the range

$$
5.5 \cdot 10^{6} \mathrm{~s} \leq \tau_{\widetilde{\tau}} \leq 1.6 \cdot 10^{9} \mathrm{~s}
$$

for the lifetime. If we restrict ourselves to stau masses above $100 \mathrm{GeV}$, the upper bound is lowered to $4.6 \cdot 10^{8} \mathrm{~s}$.

The stau abundance in the $\widetilde{G}-\widetilde{\tau}$ scenario for $\tan \beta=20$ is shown in Fig. 2. We find

$$
1.3 \cdot 10^{-13} \leq Y_{\widetilde{\tau}} \leq 6.2 \cdot 10^{-13}
$$

The abundance is smallest in those parts of the parameter space where the lightest stau masses are reached. These are the lower right corner of the bottom region and the upper border of the upper region. Conversely, we find the largest values close to the neutralino NLSP region, where $m_{\tilde{\tau}}$ is largest. Both qualitatively and quantitatively, the situation is very similar for $\tan \beta=10$, except that in this case the top $\widetilde{\tau}$ NLSP region does not exist. The approximation

$$
Y_{\widetilde{\tau}} \simeq 1.2 \cdot 10^{-13}\left(\frac{m_{\widetilde{\tau}}}{100 \mathrm{GeV}}\right)
$$




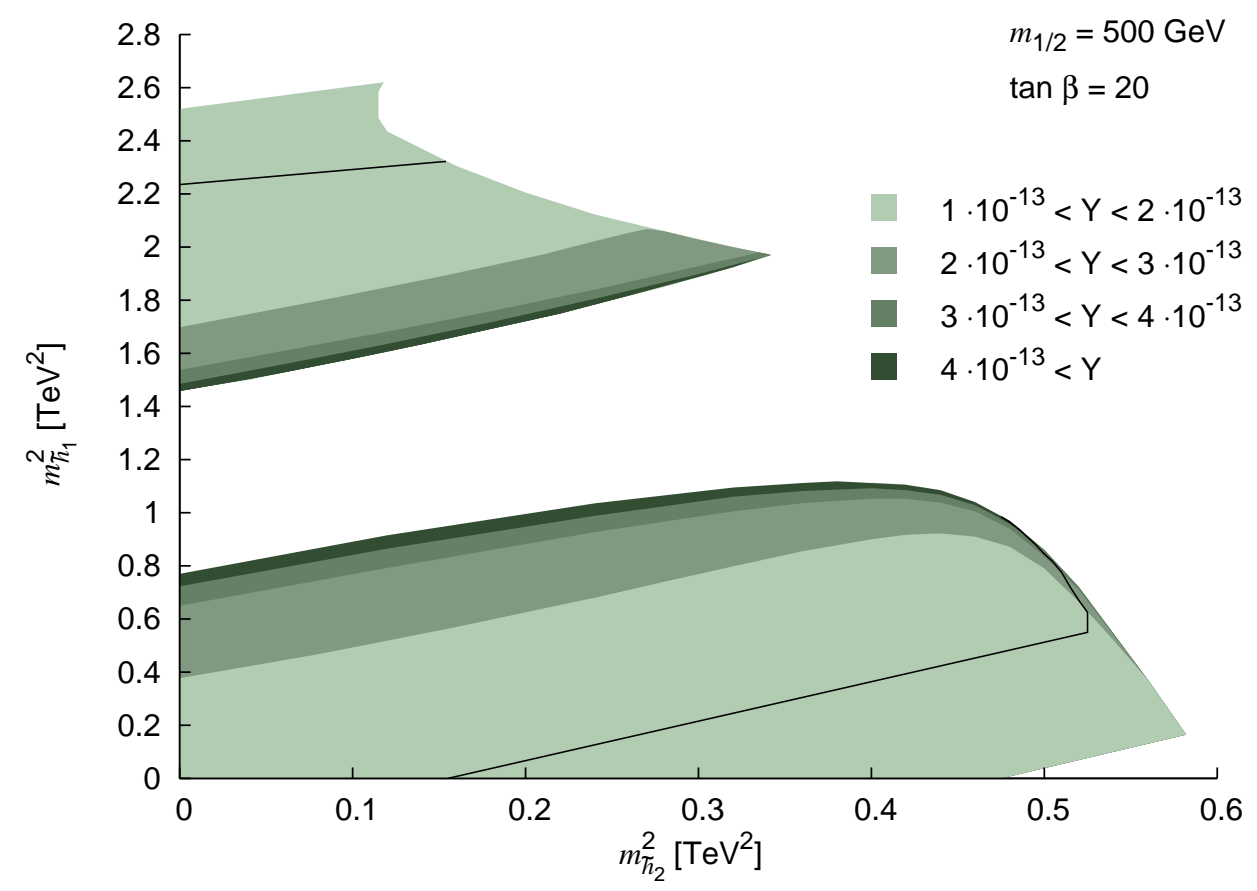

Figure 2: Stau abundance obtained numerically with micrOMEGAs in the $\widetilde{\tau}$ NLSP region. The area below (lower region) and above (upper region) the black line is excluded by the conservative electromagnetic BBN constraints for $m_{3 / 2}=50 \mathrm{GeV}$ and $\epsilon_{\mathrm{em}}=0.5 E_{\tau}$.

gives the stau abundance with a relative error of less than $10 \%$ for the largest part $(>80 \%)$ of the parameter space, where coannihilation is less important (i.e. the part not too close to the neutralino LSP region).

The average hadronic energy release $\epsilon_{\text {had }}$ from a stau decay is smaller than $10^{-2} \mathrm{GeV}$ for stau masses around $200 \mathrm{GeV}$ [17]. Consequently, in our case the combination $\epsilon_{\text {had }} Y_{\widetilde{\tau}}$ never exceeds $10^{-14} \mathrm{GeV}$, so that even the stringent hadronic BBN bound (8) is always satisfied.

However, the electromagnetic bounds are significantly more constraining. Using

$$
E_{\tau}=\frac{m_{\tilde{\tau}}^{2}-m_{3 / 2}^{2}+m_{\tau}^{2}}{2 m_{\widetilde{\tau}}}
$$

for the energy of the $\tau$ produced in the dominant two-body $\widetilde{\tau}$ decay and

$$
\epsilon_{\mathrm{em}}=x E_{\tau}
$$

for the electromagnetic energy release, we find $(\tan \beta=20)$

$$
x \cdot 3.7 \cdot 10^{-12} \mathrm{GeV} \leq \epsilon_{\mathrm{em}} Y_{\widetilde{\tau}} \leq x \cdot 5.9 \cdot 10^{-11} \mathrm{GeV} .
$$

The results for $\tan \beta=10$ fall into the same range, but with a slightly smaller spread. Here a part of the $\tau$ energy is lost to neutrinos and it is not exactly known which fraction $0.3 \leq x \leq 1$ of the energy ends up in an electromagnetic shower. 


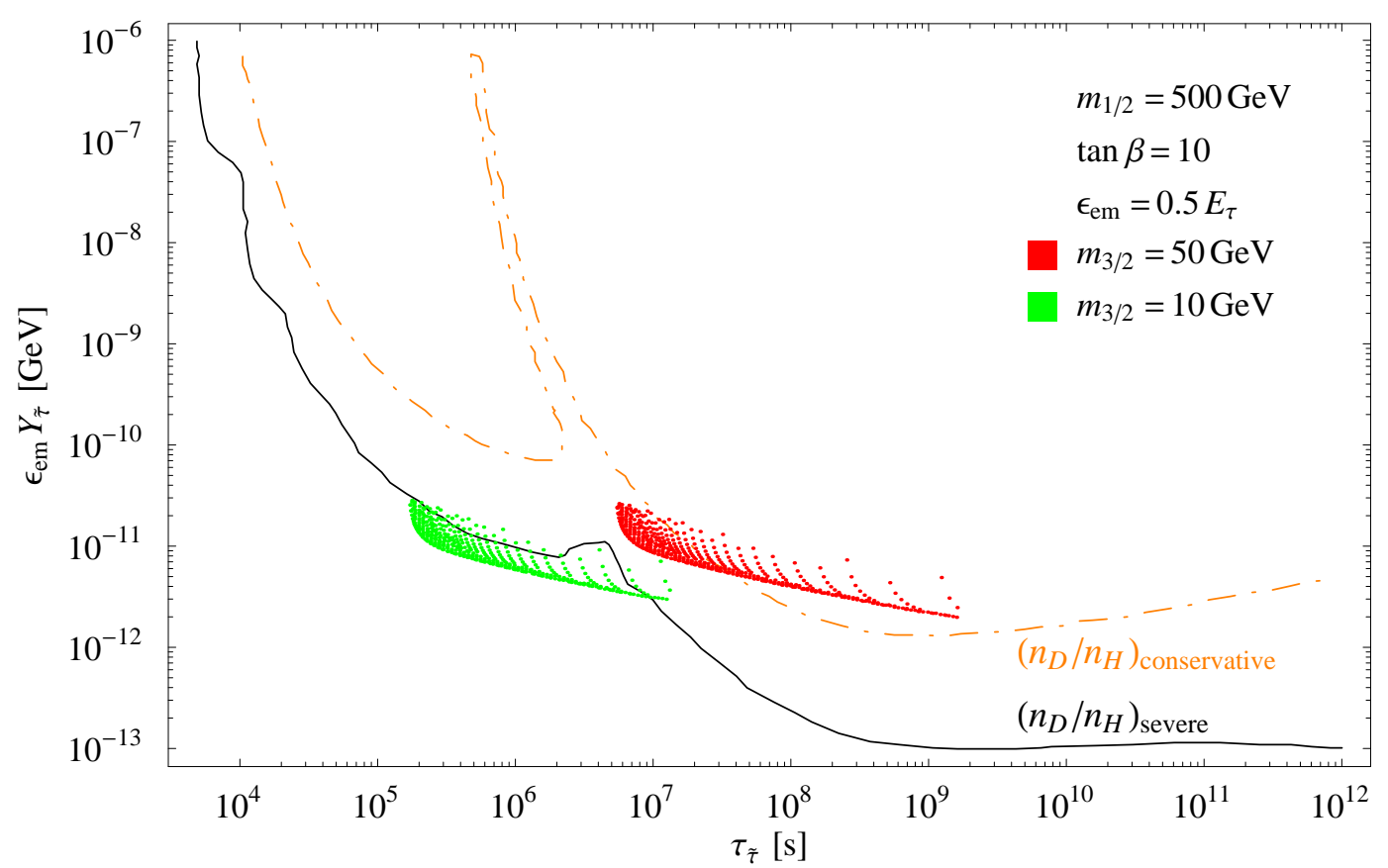

Figure 3: Points from the $\widetilde{\tau}$ NLSP region $(\tan \beta=10)$ in the $\epsilon_{\mathrm{em}} Y_{\widetilde{\tau}}-\tau_{\widetilde{\tau}}$ plane for $x=0.5$ and $m_{1 / 2}=$ $500 \mathrm{GeV}$, obtained by scanning over $m_{\tilde{h}_{1}}^{2}$ and $m_{\tilde{h}_{2}}^{2}$ with a step size of $5 \cdot 10^{-3} \mathrm{TeV}^{2}$ in both parameters. We show the results for two values of the gravitino mass, $m_{3 / 2}=50 \mathrm{GeV}$ (red or dark-gray) and $m_{3 / 2}=10 \mathrm{GeV}$ (green or light-gray). The solid black and dash-dotted orange lines show the severe and conservative electromagnetic BBN constraints from Fig. 9 of [17]. (Only the constraints derived from the deuterium abundance are shown, since those from the ${ }^{4} \mathrm{He}$ abundance are not relevant in the $\widetilde{\tau}$ NLSP region.)

In Fig. 3, we plot points from the $\widetilde{\tau}$ NLSP region for $\tan \beta=10$ in the $\epsilon_{\mathrm{em}} Y_{\widetilde{\tau}}-\tau_{\widetilde{\tau}}$ plane, assuming $x=0.5$. The red (dark-gray) points are the results for a gravitino mass of $50 \mathrm{GeV}$. We also show the severe and conservative BBN constraints from the lower plot in Fig. 9 of [17]. We find that the severe constraints are always violated, while the conservative constraints can be satisfied. The same can be seen from Fig. 2 for the case of $\tan \beta=20$, where the solid black lines mark the boundary between the parameter space regions allowed and excluded by the conservative BBN bounds. In the lower region, the area below and to the right of the line is excluded. In the upper region, this is the case for the area above the black line. Thus, it turns out that actually the largest part of the parameter space is allowed. The remaining part is typically excluded not because of an unusually large stau abundance but because of a too long lifetime due to a relatively small stau mass.

The severe BBN bounds can be satisfied, if the NLSP lifetime is shorter. This is the case for smaller gravitino masses. For $m_{3 / 2}=10 \mathrm{GeV}$, we see from the green (light-gray) points in Fig. 3 that large parts of the stau NLSP region are allowed by the severe constraints. The conservative constraints are always satisfied in this example.

Increasing the unified gaugino mass $m_{1 / 2}$ leads essentially to a rescaling of the su- 
perparticle spectrum. Since the NLSPs become heavier, their yield is larger. On the other hand, the lifetime decreases significantly, since it depends on $m_{\tilde{l}}^{5} / m_{3 / 2}^{2}$. As a consequence, a larger part of the $\widetilde{\tau}$ NLSP region is compatible with the electromagnetic BBN constraints. The hadronic constraints are still easily satisfied unless the stau mass is close to a $\mathrm{TeV}$.

Recently it has been argued that metastable charged particles alter BBN via the formation of bound states [28]. This could lead to significantly more restrictive constraints on the allowed relic abundance of these charged particles than those we considered here. In [29] bound-state effects in the CMSSM and mSUGRA are studied. The conclusion is reached that $\widetilde{\tau}$ NLSPs with lifetimes longer than $10^{3}-10^{4} \mathrm{~s}$ are excluded. If it turns out that this statement also holds in a more general framework than the CMSSM, the $\widetilde{G}-\widetilde{\tau}$ scenario will be ruled out for $m_{3 / 2} \gtrsim 10 \mathrm{GeV}$ unless there is sizeable entropy production between the decoupling of the staus from the thermal bath and the start of BBN.

\subsection{Sneutrino NLSP}

The region of sneutrino NLSP corresponds to large $m_{\tilde{h}_{1}}^{2}$ and the sneutrino masses and lifetimes lie roughly in the same range as those of the staus, but with a somewhat larger minimal mass. The relic abundance is in the narrow window

$$
1.3 \cdot 10^{-13} \leq Y_{\tilde{\nu}} \leq 4.6 \cdot 10^{-13}
$$

The BBN bounds on a sneutrino NLSP are rather weak, since the neutrinos emitted in the dominant two-body decay $\tilde{\nu} \rightarrow \nu \widetilde{G}$ interact much less than charged particles with the light nuclei. Nevertheless the very energetic anti-neutrinos (neutrinos) produced in such a decay can annihilate with the background neutrinos (anti-neutrinos) and give rise to $e^{+} e^{-}$pairs which contribute to electromagnetic showers. ${ }^{3}$ Furthermore, there are contributions from $\tilde{\nu} \rightarrow \nu \widetilde{G} \ell \bar{\ell}$, but such decays have a very small branching ratio giving $\epsilon_{\mathrm{em}}$ safely below $0.1 \mathrm{GeV}$ and are therefore negligible even for our maximal value of $Y_{\tilde{\nu}}$.

The effects of highly energetic (anti-)neutrinos on BBN have been studied in [30, 31] and [32], but in the last reference only for the specific case of an unstable gravitino decaying into sneutrino and neutrino. Assuming $\left(n_{\mathrm{D}}+n^{3} \mathrm{He}_{\mathrm{e}}\right) / n_{\mathrm{H}} \lesssim 4 \cdot 10^{-5}$ gives $Y_{X} \lesssim$ $4 \cdot 10^{-12}[31]$ for a general relic with mass around $200 \mathrm{GeV}$ and lifetime $10^{7} \mathrm{~s}$ decaying equally into neutrinos and anti-neutrinos. Shorter lifetimes are not discussed in this work, but according to [30] all constraints disappear for lifetimes shorter than $10^{6} \mathrm{~s}$, since at those earlier times high-energy photons thermalise efficiently scattering off the CMB before having the chance to interact with the light nuclei. Also the limits relax

\footnotetext{
${ }^{3}$ We expect the chemical potential of the sneutrinos to be negligible, since the lepton number asymmetry can be quickly transferred into the light leptons by the scatterings $\tilde{\nu} \tilde{\nu} \rightarrow \nu \nu, \ell \ell$. Therefore we have an equal number of sneutrinos and anti-sneutrinos, so that the NLSP decays produce both neutrinos and anti-neutrinos.
} 
for longer lifetimes, since the density of background neutrinos becomes more diluted. In [32] instead, the upper bound $\left(n_{\mathrm{D}}+n_{3^{3} \mathrm{He}}\right) / n_{\mathrm{H}} \leq 10^{-4}$ is used and the constraints are given only in the $T_{\mathrm{R}}-m_{3 / 2}$ plane. We can rephrase the strongest bound on $T_{\mathrm{R}}$ in terms of $Y_{3 / 2}$ giving

$$
Y_{3 / 2} \lesssim 10^{-12} \text { for } \tau_{3 / 2} \sim 10^{7} \mathrm{~s}
$$

Again the bound becomes quickly much weaker for longer or shorter gravitino lifetimes.

The maximal abundance (23) is an order of magnitude below the limit of [31]. We cannot directly apply the limit (24) of [32] to our case due to the different dependence on lifetime and mass in the sneutrino case, but we note that if we take the maximal bound (24) and rescale it to match the "severe" value $\left(n_{\mathrm{D}}+n_{3^{\mathrm{He}}}\right) / n_{\mathrm{H}} \leq 3.66 \cdot 10^{-5}$, we get an upper bound of $Y_{\tilde{\nu}} \leq 3.66 \cdot 10^{-13}$, which is slightly smaller than our maximal abundance. ${ }^{4}$ So electromagnetic showers in the $\widetilde{G}-\widetilde{\nu}$ scenario surely do not violate the conservative constraints, but the severe limits might become relevant for short sneutrino lifetimes. A more detailed analysis appears appropriate to draw more definite conclusions.

Let us now turn to the hadronic constraints. As for the case of the $\tilde{\tau}$ NLSP, the radiative decay producing $q \bar{q}$ pairs via an intermediate gauge boson has only a small branching ratio. The branching ratio for this decay has not yet been explicitly computed, but we can estimate it from the $\tilde{\tau}$ result. In fact the diagrams mediating the decay $\tilde{\nu}_{\mathrm{L}} \rightarrow \nu \widetilde{G} q \bar{q}$ are topologically the same as those involved in $\tilde{\tau}_{\mathrm{R}} \rightarrow \tau \widetilde{G} q \bar{q}$, apart from the fact that the intermediate gauge bosons are the $Z, W^{ \pm}$instead of $Z, \gamma$. We therefore expect for the sneutrinos a smaller hadronic branching ratio at low masses, since in both channels a massive gauge boson is involved, instead of the massless photon. In fact in the computation presented in [17] it is apparent that the $Z$ channel is strongly suppressed by phase space and remains sub-leading as long as the NLSP mass is below $200 \mathrm{GeV}$, as in our case. So we can use the $\epsilon_{\text {had }} \lesssim 10^{-2} \mathrm{GeV}$ value given for the $\tilde{\tau}$ case in [17] as a very stringent value also for the sneutrino NLSP. We obtain in this case $\epsilon_{\text {had }} Y_{\tilde{\nu}} \leq 4.6 \times 10^{-15} \mathrm{GeV}$, which is well below the hadronic limit (8).

If we compare our estimate with the conclusion in [10] that sneutrinos with masses below $400 \mathrm{GeV}$ are allowed by hadronic constraints, we find two differences that somewhat change the discussion. On the one hand, the approximate expression used in [10] to compute the sneutrino abundance always underestimates it in our case, probably due to the importance of coannihilations. On the other hand, the value of $\epsilon_{\text {had }}$ used there appears overestimated, especially for small sneutrino masses, as discussed for the stau case in [17]. The two effects partially compensate each other for small sneutrino masses.

\footnotetext{
${ }^{4}$ On general grounds the amount of $\mathrm{D}+{ }^{3} \mathrm{He}$ overproduced should be proportional to the decaying particle abundance $Y_{X}$, so we can simply rescale the constraint on $Y_{X}$ by the factor that brings their $\mathrm{D}+{ }^{3} \mathrm{He}$ abundance down to our "severe" value $3.66 \cdot 10^{-5}$. We are then imposing the bounds on Deuterium alone and not on the sum $\mathrm{D}+{ }^{3} \mathrm{He}$, which provides us with a more stringent constraint.
} 
All in all, we can conclude that the $\widetilde{G}-\widetilde{\nu}$ scenario is essentially unconstrained. The severe electromagnetic limits could be marginally relevant and are worth a more careful investigation.

\subsection{Constraints from Dark Matter and the CMB}

Gravitinos are produced non-thermally via the NLSP decays. The corresponding energy density has to be smaller than the observed cold dark matter density,

$$
\Omega_{3 / 2}^{\text {non-th }} h^{2}=\frac{m_{3 / 2}}{m_{\mathrm{NLSP}}} \Omega_{\mathrm{NLSP}}^{\mathrm{th}} h^{2} \leq \Omega_{\mathrm{DM}}<0.123 .
$$

For $m_{1 / 2}=500 \mathrm{GeV}$ and $\tan \beta=20$, we find

$$
1.8 \cdot 10^{-3} \leq \Omega_{3 / 2}^{\text {non-th }} h^{2} \leq 8.4 \cdot 10^{-3}
$$

For $\tan \beta=10$ the maximal energy density is slightly smaller. Generally, $\Omega_{3 / 2}^{\text {non-th }}$ is largest in the part of the $\widetilde{\tau}$ NLSP region which is closest to the $\chi$ LSP region. Here we also find the largest NLSP abundance (cf. Fig. 2). In the $\widetilde{G}-\widetilde{\nu}$ scenario, the maximal value for $\Omega_{3 / 2}^{\text {non-th }} h^{2}$ is just slightly smaller than in the $\widetilde{\tau}$ NLSP case.

In addition to bounds from the observed cold dark matter density there are constraints coming from distortions of the cosmic microwave background. It is well known that the CMB is very close to a Planckian distribution with zero chemical potential [33],

$$
\left.|\mu|<9 \cdot 10^{-5} \text { (at } 95 \% \text { C.L. }\right) \text {. }
$$

Since late electromagnetic energy release can lead to spectral distortions, this upper limit on $|\mu|$ can be translated into an upper limit on $\epsilon_{\mathrm{em}}$ [34]. However, this limit is based on an approximation which turned out to be reliable only for stau masses above $500 \mathrm{GeV}$ in an improved analysis [35]. For lighter staus, the bounds become weaker. As a consequence, they are less constraining than the BBN bounds in our case [17].

\subsection{Constraints on the Reheating Temperature}

At high temperatures, gravitinos are produced by thermal scatterings. The resulting energy density is approximately given by [36]

$$
\Omega_{3 / 2}^{\mathrm{th}} h^{2} \simeq 0.27\left(\frac{T_{\mathrm{R}}}{10^{10} \mathrm{GeV}}\right)\left(\frac{100 \mathrm{GeV}}{m_{3 / 2}}\right)\left(\frac{m_{\tilde{g}}}{1 \mathrm{TeV}}\right)^{2},
$$

where $m_{\tilde{g}}$ is the running gluino mass evaluated at low energy. For $m_{1 / 2}=500 \mathrm{GeV}$, we have $m_{\tilde{g}} \simeq 1150 \mathrm{GeV}$. The maximal possible reheating temperature $T_{\mathrm{R}}$ is obtained for the heaviest allowed gravitino mass. In the $\widetilde{G}-\widetilde{\tau}$ scenario, the gravitino mass is strongly constrained by BBN. The largest allowed value is around $m_{3 / 2} \simeq 70 \mathrm{GeV}$. Using this 
upper limit and taking as lower bound $m_{3 / 2}=10 \mathrm{GeV}$, we can calculate an allowed range for the reheating temperature, assuming that all the dark matter is made up of gravitinos. Since the non-thermal contribution is negligible (cf. Eq. (26)), one obtains from $\Omega_{3 / 2}^{\text {th }} \leq \Omega_{\mathrm{DM}}$ and Eq. (7)

$$
3 \cdot 10^{8} \mathrm{GeV} \lesssim T_{\mathrm{R}} \lesssim 3 \cdot 10^{9} \mathrm{GeV}
$$

This is marginally compatible with the minimal temperature required for thermal leptogenesis [37]. Increasing the unified gaugino mass $m_{1 / 2}$ essentially leads to a rescaling of the gluino and gravitino masses, which lowers the upper bound on $T_{R}$. Therefore a small gaugino mass is needed for a high reheating temperature.

The $\widetilde{G}-\widetilde{\nu}$ scenario is less constrained by BBN and therefore allows for a much heavier gravitino. The only restriction is that the gravitino be lighter than the sneutrino, $m_{3 / 2} \lesssim$ $200 \mathrm{GeV}$. This leads to a larger allowed range for the reheating temperature,

$$
3 \cdot 10^{8} \mathrm{GeV} \lesssim T_{\mathrm{R}} \lesssim 7 \cdot 10^{9} \mathrm{GeV}
$$

which is consistent with thermal leptogenesis.

\section{Conclusions}

We have discussed dark matter candidates in theories with gaugino-mediated supersymmetry breaking and compact dimensions of order the unification scale. Varying the boundary conditions for bulk Higgs fields at the unification scale, at different points in parameter space, the gravitino, a neutralino or a scalar lepton can be the lightest or next-to-lightest superparticle.

We have investigated constraints from the observed dark matter density and primordial nucleosynthesis on the different scenarios. The resulting viable dark matter candidates in gaugino mediation are summarised in Fig. 4. A neutralino LSP as the dominant component of dark matter is a viable possibility. Gravitino dark matter with a $\widetilde{\tau}$ NLSP is consistent for a wide range of parameters only with the "conservative" BBN constraints (cf. Eqs. (4)). The "severe" BBN bounds (cf. Eqs. (3)) require either a gravitino mass close to the lower bound in gaugino mediation or entropy production after $\widetilde{\tau}$ decoupling. Gravitino dark matter with a $\widetilde{\nu}$ NLSP can also be realised and is essentially unaffected by all constraints.

\section{Acknowledgements}

We would like to thank Genevieve Bélanger, Nicolao Fornengo, Koichi Hamaguchi, Jan Hamann and Frank Steffen for valuable discussions. WB thanks the Galileo Galilei 

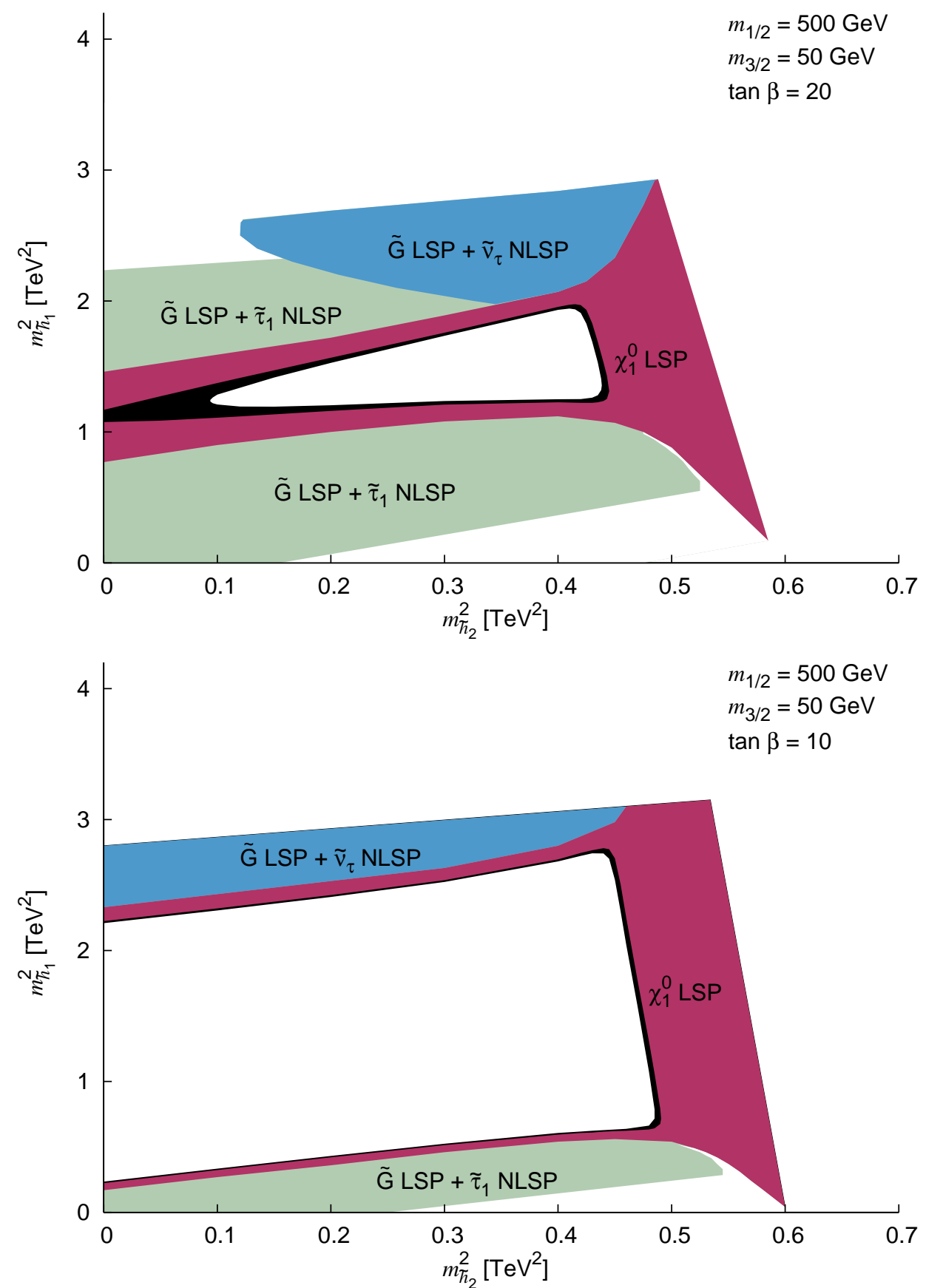

Figure 4: Allowed parameter space for the soft Higgs masses in gaugino mediation. In the black and magenta (dark-gray) coloured regions a neutralino is the LSP, whereas in the green (light-gray) and blue (medium-gray) regions the gravitino is the LSP with either a stau or a sneutrino being the NLSP. All white areas are excluded by bounds from the observed dark matter density and the "conservative" constraints from primordial nucleosynthesis. 
Institute for Theoretical Physics for the hospitality and the INFN for partial support during the completion of this work. This work has been supported by the "Impuls- und Vernetzungsfonds" of the Helmholtz Association, contract number VH-NG-006.

\section{References}

[1] D. E. Kaplan, G. D. Kribs, M. Schmaltz, Phys. Rev. D62 (2000), 035010 [hep$\mathrm{ph} / 9911293]$.

[2] Z. Chacko, M. A. Luty, A. E. Nelson, E. Ponton, JHEP 01 (2000), 003 [hep$\mathrm{ph} / 9911323]$.

[3] J. R. Ellis, C. Kounnas, D. V. Nanopoulos, Nucl. Phys. B247 (1984), 373; K. Inoue, M. Kawasaki, M. Yamaguchi, T. Yanagida, Phys. Rev. D45 (1992), 328.

[4] W. Buchmüller, J. Kersten, K. Schmidt-Hoberg, JHEP 02 (2006), 069 [hep$\mathrm{ph} / 0512152]$.

[5] T. Falk, K. A. Olive, M. Srednicki, Phys. Lett. B339 (1994), 248 [hep-ph/9409270].

[6] Particle Data Group, S. Eidelman, et al., Phys. Lett. B592 (2004), 1.

[7] W. Buchmüller, K. Hamaguchi, J. Kersten, Phys. Lett. B632 (2006), 366 [hep$\mathrm{ph} / 0506105]$.

[8] W. Buchmüller, K. Hamaguchi, M. Ratz, T. Yanagida, Phys. Lett. B588 (2004), 90 [hep-ph/0402179]; K. Hamaguchi, Y. Kuno, T. Nakaya, M. M. Nojiri, Phys. Rev. D70 (2004), 115007 [hep-ph/0409248]; J. L. Feng, B. T. Smith, Phys. Rev. D71 (2005), 015004 [hep-ph/0409278]; A. Brandenburg, L. Covi, K. Hamaguchi, L. Roszkowski, F. D. Steffen, Phys. Lett. B617 (2005), 99 [hep-ph/0501287]; H.-U. Martyn, hep-ph/0605257; J. R. Ellis, A. R. Raklev, O. K. Øye, hep-ph/0607261.

[9] J. L. Feng, A. Rajaraman, F. Takayama, Phys. Rev. D68 (2003), 063504 [hepph/0306024]; M. Fujii, M. Ibe, T. Yanagida, Phys. Lett. B579 (2004), 6 [hep$\mathrm{ph} / 0310142]$.

[10] J. L. Feng, S. Su, F. Takayama, Phys. Rev. D70 (2004), 075019 [hep-ph/0404231].

[11] J. R. Ellis, K. A. Olive, Y. Santoso, V. C. Spanos, Phys. Lett. B588 (2004), 7 [hep-ph/0312262]; L. Roszkowski, R. Ruiz de Austri, K.-Y. Choi, JHEP 08 (2005), 080 [hep-ph/0408227].

[12] T. Asaka, W. Buchmüller, L. Covi, Phys. Lett. B563 (2003), 209 [hep-ph/0304142]. 
[13] Z. Chacko, M. A. Luty, E. Ponton, JHEP 07 (2000), 036 [hep-ph/9909248].

[14] R. H. Cyburt, J. R. Ellis, B. D. Fields, K. A. Olive, Phys. Rev. D67 (2003), 103521 [astro-ph/0211258].

[15] M. Kawasaki, K. Kohri, T. Moroi, Phys. Rev. D71 (2005), 083502 [astro$\mathrm{ph} / 0408426]$.

[16] K. Jedamzik, hep-ph/0604251.

[17] F. D. Steffen, JCAP 09 (2006), 001 [hep-ph/0605306].

[18] G. Belanger, F. Boudjema, A. Pukhov, A. Semenov, Comput. Phys. Commun. 149 (2002), 103 [hep-ph/0112278]; G. Belanger, F. Boudjema, A. Pukhov, A. Semenov, Comput. Phys. Commun. 174 (2006), 577 [hep-ph/0405253].

[19] B. C. Allanach, Comput. Phys. Commun. 143 (2002), 305 [hep-ph/0104145].

[20] CDF and D0 Collaborations, Tevatron Electroweak Working Group, hepex/0603039.

[21] T. Jittoh, J. Sato, T. Shimomura, M. Yamanaka, Phys. Rev. D73 (2006), 055009 [hep-ph/0512197].

[22] U. Seljak, A. Slosar, P. McDonald, JCAP 0610 (2006), 014 [astro-ph/0604335].

[23] M. E. Gomez, T. Ibrahim, P. Nath, S. Skadhauge, Phys. Rev. D70 (2004), 035014 [hep-ph/0404025]; J. R. Ellis, S. Heinemeyer, K. A. Olive, G. Weiglein, JHEP 02 (2005), 013 [hep-ph/0411216]; A. Djouadi, M. Drees, J.-L. Kneur, JHEP 03 (2006), 033 [hep-ph/0602001].

[24] N. Fornengo, private communication.

[25] CDMS Collaboration, D. S. Akerib, et al., Phys. Rev. Lett. 96 (2006), 011302 [astro-ph/0509259].

[26] CDMS-II Collaboration, D. A. Bauer, AIP Conf. Proc. 815 (2006), 105.

[27] D. G. Cerdeño, K.-Y. Choi, K. Jedamzik, L. Roszkowski, R. Ruiz de Austri, JCAP 0606 (2006), 005 [hep-ph/0509275].

[28] M. Pospelov, hep-ph/0605215; K. Kohri, F. Takayama, hep-ph/0605243;

M. Kaplinghat, A. Rajaraman, astro-ph/0606209.

[29] R. H. Cyburt, J. Ellis, B. D. Fields, K. A. Olive, V. C. Spanos, astro-ph/0608562. 
[30] J. Gratsias, R. J. Scherrer, D. N. Spergel, Phys. Lett. B262 (1991), 298.

[31] A. A. de Laix, R. J. Scherrer, Phys. Rev. D48 (1993), 562.

[32] M. Kawasaki, T. Moroi, Phys. Lett. B346 (1995), 27 [hep-ph/9408321].

[33] D. J. Fixsen, et al., Astrophys. J. 473 (1996), 576 [astro-ph/9605054].

[34] W. Hu, J. Silk, Phys. Rev. D48 (1993), 485.

[35] R. Lamon, R. Durrer, Phys. Rev. D73 (2006), 023507 [hep-ph/0506229].

[36] M. Bolz, A. Brandenburg, W. Buchmüller, Nucl. Phys. B606 (2001), 518 [hepph/0012052]; J. Pradler, F. D. Steffen, hep-ph/0608344.

[37] W. Buchmüller, P. Di Bari, M. Plümacher, Ann. Phys. 315 (2005), 305 [hepph/0401240]. 